\title{
Single-Step Synthesis of Dual Phase Bright Blue-Green Emitting Lead Halide Perovskite Nanocrystal Thin Films
}

\author{
Harshita Bhatia ${ }^{1}$, Julian A. Steele ${ }^{2}$, Cristina Martin ${ }^{1,3}$, Masoumeh Keshavarz ${ }^{1}$, Guillermo Solis- \\ Fernandez ${ }^{1}$, Haifeng Yuan ${ }^{1}$, Guillaume Fleury ${ }^{2}$, Haowei Huang ${ }^{2}$, Iurii Dovgaliuk ${ }^{4}$, Dmitry Cher- \\ nyshov $^{4}$, Jelle Hendrix ${ }^{1,5}$, Maarten B.J. Roeffaers ${ }^{2}$, Johan Hofkens ${ }^{1^{*}}$, Elke Debroye ${ }^{{ }^{*}}$ \\ 1 Department of Chemistry, KU Leuven, Celestijnenlaan 200F, B-3001 Leuven, Belgium \\ 2 Department of Microbial and Molecular Systems, Centre for Surface Chemistry and Catalysis, KU Leuven, B-3001 \\ Leuven, Belgium \\ 3 Departamento de Química Física, Facultad de Farmacia, Universidad de Castilla-La Mancha, 02071 Albacete, Spain \\ 4 Swiss-Norwegian Beamlines at the European Synchrotron Radiation Facility, 71 avenue des Martyrs, F-38ooo Gre- \\ noble, France
}

5 Dynamic Bioimaging Lab, Advanced Optical Microscopy Centre and Biomedical Research Institute, Hasselt University, Agoralaan C (BIOMED), B359o Diepenbeek.

\begin{abstract}
Metal trihalide perovskites are rapidly redefining the landscape of solid-state semiconductors utilized as active medium in photovoltaics and in light generation. Within this materials space, organic-inorganic hybrid formamidinium lead bromide ( $\mathrm{FAPbBr}_{3}$ ) has arisen as a promising candidate for efferent light emitting devices, due to its capacity for sharp and bright green light emissions $(530 \mathrm{~nm})$. Herein we have applied a facile single-step ligand-mediated method for phase-controlled synthesis of $\mathrm{FAPbBr}_{3}$ cube- and rod-shaped nanocrystals (NCs), starting from different ratios of precursor agents. Examining their structural and optoelectronic properties - using a combination of synchrotron X-ray diffraction, X-ray spectroscopy, scanning electron microscopy and steady-state and time-resolved photoluminescence (PL) - we reveal the two NC types to fundamentally differ. While the cube-shaped NCs exhibit properties aligning with that of bulk $\mathrm{FAPbBr}_{3}$, the nanorods exhibit a two-phase microstructure and the co-existence of both a typical cubic perovskite structure alongside the formation of a new low-symmetry monoclinic phase $\left(\mathrm{P}_{2} / \mathrm{m}\right)$. Further, the two-phase nanorods display a bright dual PL emission (peaks centered near $490 \mathrm{~nm}$ and $530 \mathrm{~nm}$ ) and complex luminescence dynamics, properties characteristic of quasi-2D perovskites. The two phase nanorods generation can be assigned to the proton exchange in the presence of excess of $\mathrm{FA}^{+}$during the synthesis.
\end{abstract}

Very few materials within optoelectronics research have garnered the scientific and technological interest generated by organic-inorganic hybrid perovskite semiconductors - possessing the general $\mathrm{ABX}_{3}$ chemical formula. ${ }^{1,2}$ This recent spike in attention for trihalide perovskite is owed to their unique and interesting combination of optoelectronic properties; from high absorption coefficients and long exciton diffusion lengths (100-10oo $\mathrm{nm}$ ), to small exciton binding energies and high quantum yields. ${ }^{3}$ Further, their tunable emission retains extremely high color purity, all while being solution processable. Needless to say, these properties combine to lay a promising path toward high performance and low-cost light emitting diodes (LEDs). ${ }^{4-6}$
Even though various perovskite systems have shown photoluminescence quantum yields (PLQYs) up to $90 \%$ at $450 \mathrm{~nm},{ }^{7,8}$ producing color stable and efficient blue LEDs remains challenging. ${ }^{9-12}$ Another critical issue is the poor stability of perovskite thin films in LED devices. ${ }^{13,14} \mathrm{Im}-$ portantly, the application of polycrystalline material should be avoided since it contains a large number of intrinsic defects which are detrimental for the charge carrier dynamics and initiate parasitic non-radiative recombination pathways. ${ }^{14,15}$ The PL efficiency can be improved by confining excitons in small nanograins ( 100 $\mathrm{nm}),{ }^{4,15}$ or by making use of quantum dots $(<20 \mathrm{~nm})$ or nanocrystals while simultaneously passivating the surface defects using ligands ${ }^{16,17}$ Recently, a new perovskite material class has emerged, known as quasi-2D perovskites, 
possessing the merits of both $3 \mathrm{D}$ and $2 \mathrm{D}$ structures. ${ }^{18-20}$ Quasi-2D systems are formed by incorporating bulky insulating cation spacers into the inorganic sheets of corner sharing octahedra, in which the insulating cation acts as a barrier and the inorganic layer as a well. Therefore, the generated excitons are confined in the natural multiple-quantum well structure, giving rise to superior PL properties than those of $3 \mathrm{D}$ counterparts. ${ }^{21}$

In this manuscript, we report on the synthesis of brightly emissive $\mathrm{FAPbBr}_{3}$ nanocrystals (NCs), using a facile and scalable ligand-mediated wet-chemical method under ambient conditions. ${ }^{22-24}$ By taking advantage of proton exchange processes between alkylamines during growth, we find that by increasing the molar concentration of FABr relative to that of the $\mathrm{PbBr}_{2}$ precursor (from one to three-fold), the formation of NCs with a rod-like structure is promoted over nanocubes. An extensive photophysical evaluation of the NCs reveals that, unlike the conventional properties of the nanocubes, our nanorods, $\mathrm{FA}_{2} \mathrm{PbBr}_{4}-$ $\mathrm{FAPbBr}_{3}$, thin films exhibit characteristics consentient with recent reports of quasi-2D perovskite systems, displaying a blue-green emission ( $490 \mathrm{~nm}$; PLQY $32 \%$ at 445 $\mathrm{nm})$, originating from the presence of a relatively exotic monoclinic $\mathrm{FAPbBr}_{3} \mathrm{NC}$ phase.

Results and Discussion
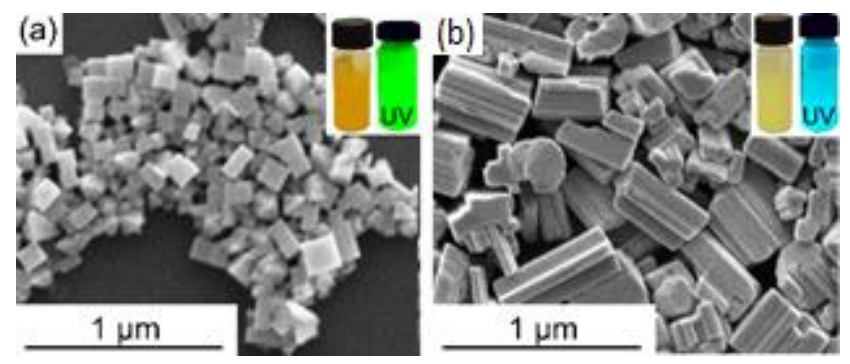

Figure 1. SEM images of (a) $\mathrm{FAPbBr}_{3}$ nanocubes $\left(\mathrm{PbBr}_{2}: \mathrm{FABr}\right.$ $=1: 1)$ and (b) $\mathrm{FAPbBr}_{3}$ nanorods $\left(\mathrm{PbBr}_{2}: \mathrm{FABr}=1: 3\right)$. The insets picture the suspensions under visible (right) and 365 nm UV (left) illumination.

Preparation of NCs:

First we outline a new and facile single-step, high yielding route for the synthesis of quasi-2D perovskites. Interestingly, unlike other reported procedures wherein the ammonium cation is intentionally added to obtain the quasi$2 \mathrm{D}$ structure, ${ }^{5,25,26}$ our synthesis approach induces the formation of an ammonium cation during the synthesis. The preparation steps involve a ligand-mediated wetchemical approach which is conducted under ambient conditions. ${ }^{22-24,27}$ Specifically, by oversaturating the molar concentration of $\mathrm{FABr}$ relative to that of the $\mathrm{PbBr}_{2}$ precursor from 1:1 to 3:1, proton exchange between the alkylamines is believed to substantially influence the perovskite formation, i.e. the formed protonated alkylamine capping ligand is found to be integrated into the perovskite lattice. Note that further support and discussion on this process is still to come (vide infra, section XPS, page4). Ultimately, on varying the precursors' molar ratio, colloidal solutions appear distinct in color and possess a substantial difference in size and morphology of the NCs (Figure 1 and $S_{2}$ ); green emitting nanocubes (with typical orange color) on the order of $200 \mathrm{nms}$ are formed with a $\mathrm{PbBr}_{2}$ : $\mathrm{FABr}$ ratio of 1:1, while larger blue emitting nanorods (yellow in color) with dimensions in the order of 400 by $70 \mathrm{~nm}$ are formed with a precursor ratio of 1:3. Before characterization, the NCs are transferred from solution onto glass substrates to form stable NC thin films.

\section{Structural Properties}

We begin with a structural and compositional overview of the different orange and yellow $\mathrm{FAPbBr}_{3} \mathrm{NCs}$ presented in Figure 1. From here on we simply refer to the two NC types based on their differing morphologies, i.e. nanocubes and nanorods, whereby the $\mathrm{FAPbBr}_{3}$ nanocubes will act as an archetypal control to the relatively exotic properties of the nanorods.

\section{EDX compositional analysis:}

On the outset, we find that the two NC types differ not only in their color with and without UV excitation (Figure 1), but also in their fundamental compositions. Specifically, surface sensitive EDX analysis (Figure $\mathrm{S}_{3}$ ) revealed that the nanocubes possess the nominal $1: 3 \mathrm{~Pb} / \mathrm{Br}$ atomic ratio for a $3 \mathrm{D}$ metal trihalide perovskite. In contrast, an average atomic ratio of $1: 4$ is recorded from the nanorods, deviating with a statistical relevance from the nominal 1:3 value seen in the nanocubes. These findings indicate that an excess of $\mathrm{FABr}$ induces two distinct phenomena: a transformation in morphology (i.e. cubes to rods) and an increase in $\mathrm{Br}$ content relative to $\mathrm{Pb}$. We point out that such compositional deviations are consistent with several recent reports on $2 \mathrm{D}$ perovskite systems. ${ }^{28,29}$

\section{Synchrotron X-ray diffraction:}

Next we exact the crystal structure and phase purity of the $\mathrm{FAPbBr}_{3} \mathrm{NCs}$ using high-resolution XRD. The two NC types were placed in quartz capillaries exposed to a high- 

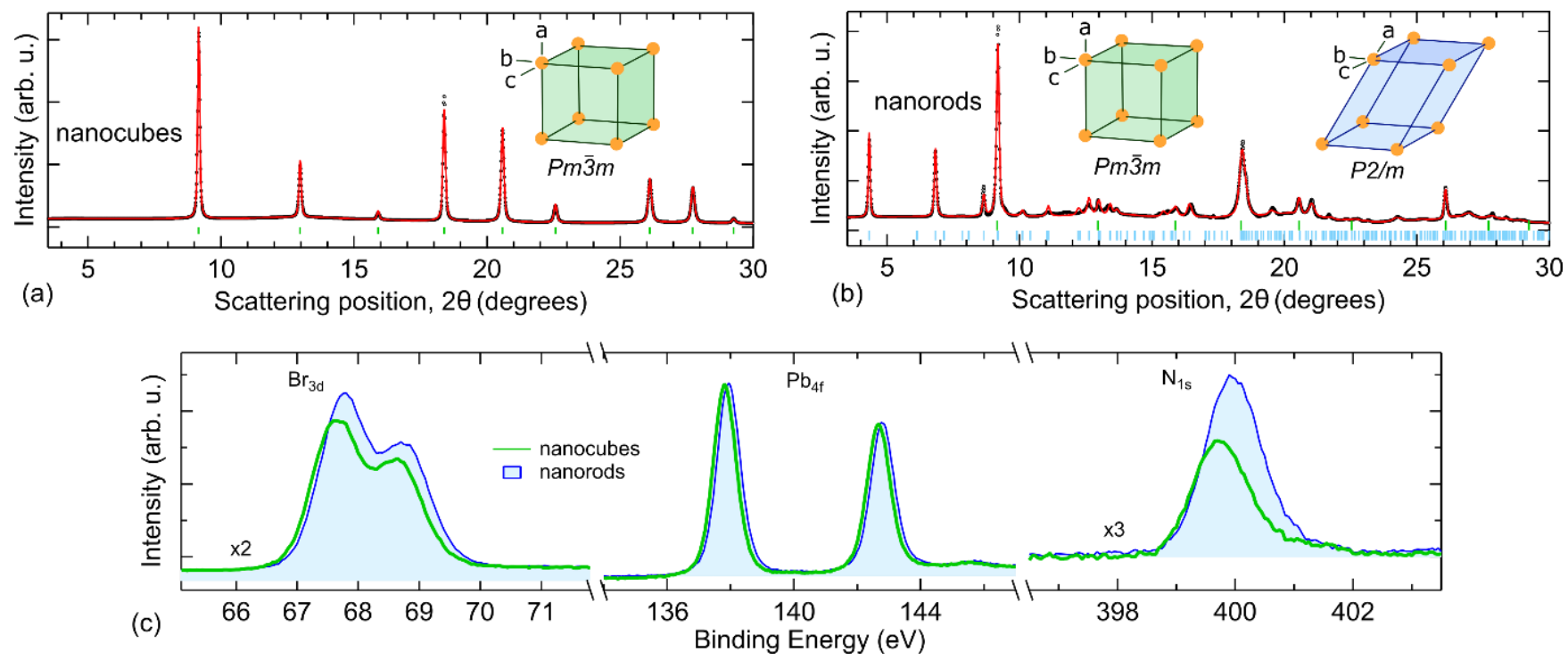

Figure 2. Powder XRD patterns of $\mathrm{FAPbBr}_{3}$ a) nanocubes and b) nanorods (black circles) and their respective refinements (red line). The respective insets show the different unit cells used to generate the refinement, corresponding to the peak positions at

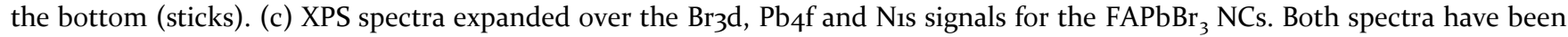
normalized to their respective Pb4f peaks (at $137 \mathrm{eV}$ ). The peak analysis can be found in Table S1 of SI.

throughput synchrotron X-ray beam tuned to a wavelength of $0.95774 \AA$, under ambient conditions. Figure 2 presents the respective diffraction scans recorded and the structural refinements made for the two types of NCs. First, in line with the well-known thermodynamically favored structure of $\mathrm{FAPbBr}_{3},{ }^{30,31}$ the nanocubes exhibit a cubic structure (refined to $\mathrm{a}=5.9943 \AA$ using the $\mathrm{Pm} \overline{\mathrm{m}}$ space group) with no obvious reduction in the unit cell translational symmetry. ${ }^{32}$ Again, this is in strong contrast to the diffraction pattern recorded for the yellow $\mathrm{FAPbBr}_{3}$ nanorods; here we see the introduction of a large number of additional Bragg peaks, along with a substantial reduction in symmetry, suggested by the extensive splitting of the cubic-like scattering features. A refinement of the XRD data in Figure $2 b$ is best made accounting for two separate phases. Namely, a low-symmetry monoclinic structure $(\mathrm{a}=9.0060 \AA$, b = 8.0570 $\AA, c=12.7940 \AA$ and $\beta=95.40^{\circ}$ using the $\mathrm{P}_{2} / \mathrm{m}$ space group) is derived from the collection of smaller peaks scattering at both low and high angles, while a smaller contribution from the nominal ${ }_{3} \mathrm{D}$ cubic phase is also present. While the refined XRD scan presented in Figure $2 \mathrm{~b}$ is quite agreeable to the experimental data, exacting further details is made difficult by the strong presence of a (100) texture (indicative of the rod-shaped morphology), complex organics (i.e. FA), and the twophase character. To the best of our knowledge, this is the first time that $\mathrm{FAPbBr}_{3}$ has been found to exist in a monoclinic phase. In combination with the compositional difference revealed through the prior EDX data, the monoclinic phase determination can be explained by the formation of a quasi-2D $\mathrm{FAPbBr}_{3}$ structure. ${ }^{33}$ The monoclinic cell can be considered as a distorted version of $\sqrt{2} a_{c} \times \sqrt{2} a_{c} \times 2 a_{c}$ orthorhombic cell. Such a lattice transformation in perovskites is an indication of a cooperative tilting of $\mathrm{PbBr}_{6}$ octahedra around c-axis; opposite tilting direction for two neighboring layers doubles the c-axis. ${ }^{34}$ Notably a and b pseudo-cubic cell dimensions are shorter than $\mathrm{c}$ and on average, are practically the same as for the cubic phase (6.364x5.697x6.397, $\left.0.5^{*}(6.364+5.697)=6.03, a_{c}=5.9943\right)$. We point out that the low-angle monoclinic peaks in Figure $2 \mathrm{~b}$ do resemble the interplanar scattering peaks seen in true $2 \mathrm{D}$ crystal planes, ${ }^{35}$ however, such a feature is not necessarily synonymous in a quasi-2D arrangement. The compositional difference revealed through the prior EDX data, agree with the formation of a quasi-2D FAPbBr3 layers. ${ }^{33}$ Although, unit cell dimensions clearly indicate that ${ }_{3} \mathrm{D}$ framework of $\mathrm{PbBr}_{6}$ corner-sharing octahedra is preserved.

\section{X-ray photoelectron spectroscopy (XPS):}

In the absence of clear low-angle interplanar $2 \mathrm{D}$ XRD peaks, we now explore the different coordination details of NCs within the context of the anticipated quasi-2D system (nanorods). Figure $2 \mathrm{c}$ presents the X-ray photoelectron spectroscopy (XPS) data recorded from thin films made of the two NCs. First, the spectra acquired from both samples possess consistent chemical features; distinctive peaks of $\mathrm{Br}_{3} \mathrm{~d}(\sim 67.5$ and $68.5 \mathrm{eV}), \mathrm{Pb}_{4} \mathrm{f}$ $(\sim 137.8$ and $142.7 \mathrm{eV})$ and Nis $(\sim 399.8 \mathrm{eV})$. Carbon peaks were also recorded from these samples, though interpreting their XPS signals is made difficult by the choice of carbon tape sample support. The small difference in peak positions $(<0.2 \mathrm{eV})$ and widths all fall well within the experimental error of the measurement (induced by crystal charging, etc.), with no notable chemical shifts or 

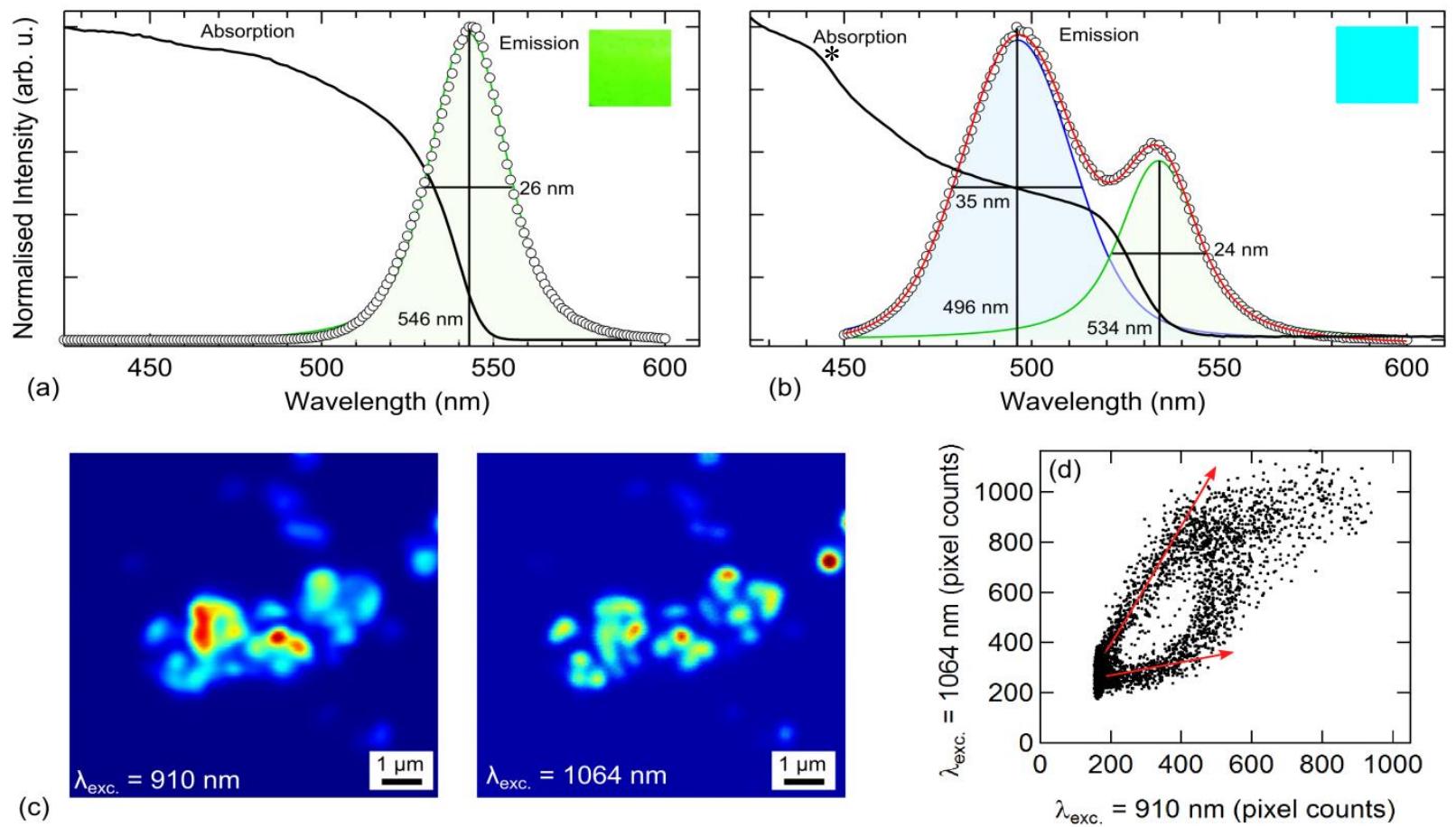

Figure 3. Normalized absorption and emission spectra of dropcasted thin films of FAPbBr3 (a) nanocubes and (b) nanorods, prepared using 1:1 and 1:3 $\mathrm{PbBr}$ /FABr precursor ratio, respectively. For the nanorods, the second high-energy excitonic absorption feature is identified by ' ' '. The colored squares inset to both plots reflect the difference in perceived PL emission color under a $365 \mathrm{~nm}$ UV lamp. (c) Corresponding two-photon PL maps of a collection of FAPbBr3 nanorods, using two $910 \mathrm{~nm}$ (left) and $1064 \mathrm{~nm}$ (right) photons to excite the emission signals. In both cases, photoluminescence is detected in a reduced spectral window ranging from 495-540 nm, which covers most of the PL emission. (d) Correlation plot of each corresponding map pixels shown in (c), where the red arrows provide a guide for the eye to highlight the anti-correlation.

oxidation states present. Notably in Figure 2c, with both spectra normalized to the $\mathrm{Pb}_{4} \mathrm{f}$ peak at $137.8 \mathrm{eV}$ there exists a large differencing in the intensity of signal detected from both elements $\mathrm{Br}$ and $\mathrm{N}$; in both cases, these signals are relatively stronger with a move to the higher $\mathrm{FAPb}$ molar fraction, i.e. the synthesis of quasi-2D nanorods. For completeness, we provide the peak analysis in Table $\mathrm{S} 1$ of the SI. This observed rise in the $\mathrm{Br}$ signal is consistent with the previous EDX studies, confirming that the $\mathrm{Br} / \mathrm{Pb}$ ratio in the nanorods increased relative to the nanocubes. We again assign this increase to the highly distorted monoclinic perovskite phase detected through XRD analysis in Figure $2 \mathrm{a}$, and is consentient with the compositional properties commonly observed in $2 \mathrm{D}$ perovskite structures; with the formula $\left(\mathrm{RNH}_{3}\right)_{2} \mathrm{PbBr}_{4}$. In addition, the large increase in the $\mathrm{N} / \mathrm{Pb}$ ratio detected within the XPS signals further supports the notion of excess formamidinium and oleylammonium cations, participating at the quasi-2D perovskite surface. ${ }^{36-39}$ Note that the abundance of $\mathrm{Br}$ atoms at the perovskite surface is anticipated to play an important role in potentially passivating parasitic surface states. ${ }^{40}$

In the presence of an excess of formamidinium, proton exchange can occur within amine-based systems via homoconjugation $\left(\mathrm{FAH}^{+} \cdots \mathrm{OAm}\right){ }^{41}$ It follows that oleylamine is transformed into the oleylammonium cation, which competes with $\mathrm{FA}^{+}$ions for lattice sites

and promotes the formation of low-dimensional structures represented by the formula $\left[\mathrm{RNH}_{3}\right]_{2}\left[\mathrm{FAPbBr}_{3}\right]_{\mathrm{n}-1} \mathrm{PbBr}_{4} \cdot{ }^{42,43}$ As mentioned above, our synthesis approach induces the formation of the ammonium cation during the synthesis. To probe the mechanism involved here, experiments targeting the role of proton excess were carried out. Figure $\mathrm{S}_{4}$ displays the SEM and XRD characterization of materials grown when $\mathrm{HBr}$ was added to the synthesis protocol with a $\mathrm{PbBr}_{2} / \mathrm{FABr}$ 1:1 percursor ratio. In this case, the excess protons provided by the $\mathrm{HBr}$ result in the formation of rod-like $\mathrm{FAPbBr}_{3} \mathrm{NCs}$ with again the contribution of a monoclinic crystal structure. Therefore, $\mathrm{HBr}$ is anticipated to form as an intermediate product during the reaction of $\mathrm{PbBr}_{2}$ with a higher molar concentration of FABr.

\section{Photophysical Properties}

Next we evaluate the all-important optical properties of the $\mathrm{FAPbBr}_{3} \mathrm{NCs}$ drop-casted in the form of a thin film. Figure 3 shows the steady-state absorption - reflectance spectra converted using the Kubelka-Munk equation $\alpha(R)=(1-R)^{2} /(2 R)$ and PL emission spectra of the FAP- 
$\mathrm{bBr}_{3}$ nanocubes and nanorods. Both samples exhibit a wide absorption band and intense PL emission, however there are several important differences. For the nanocubes, the UV-VIS absorption spectrum in Figure 3a is typical of $\mathrm{FAPbBr}_{3}$, with an absorption onset near $600 \mathrm{~nm}$ together with an excitonic feature near $530 \mathrm{~nm}$. The sharp and intense PL emission is also characteristic, ${ }^{30,44}$ centered at $546 \mathrm{~nm}$ and with a narrow full width at half-maximum (FWHM) of $26 \mathrm{~nm}$, indicative of the high color purity of $\mathrm{FAPbBr}_{3}$, and confirms the overall high quality and uniformity of the nanocubes. Furthermore, the thin film made from the nanocubes is found to remain stable and retain their bright emission, achieving a PL efficiency of $30 \%$ at 485 excitation wavelength.

On the other hand, the absorption data recorded from the nanorods contain two clear transition features in Figure $3 \mathrm{~b}$, consistent within with a two-phase quasi-2D framework, each having a different electronic structure and bandgap. While the excitonic band at $522 \mathrm{~nm}$ is again resolved in the nanorods - characteristic of a $3 \mathrm{D}$ $\mathrm{FAPbBr}_{3}$ direct bandgap - an additional blue-shifted excitonic band appears at $440 \mathrm{~nm}$ (identified here by '*'). Relative to the nanocubes, the shift in absorption to lower wavelengths in the nanorods specifies its shift in visible color, from orange to yellow, as shown in Figure 1. Although the deformation of a cubic unit cell to a lowsymmetry perovskite (like the monoclinic phase revealed here) is typically paralleled by only a minor shift in the electronic bandgap, ${ }^{40}$ the blue-shift expressed in our data is inexplicably large and cannot be accounted for based solely on a phase restructuring. Such a strong blue-shift is in agreement with a low-dimensional perovskite structure, consisting of just a few layers of perovskite unit cells $s^{45}$ and is indicative of quantum confinement. ${ }^{46}$ The deconvoluted PL spectrum of the nanorods exhibits a notably more complex emission lineshape than the nanocubes; two relatively narrow PL peaks appear, one centered at $496 \mathrm{~nm}$ and another at $530 \mathrm{~nm}$. The low-energy emission corresponds well to the excitonic peak in the absorption spectrum at 520 $\mathrm{nm}$, representing the emission from the $3 \mathrm{D}$ portion of the NC composite. Conversely, we assign the highenergy blue emission to radiative recombination originating from the quasi-2D components. ${ }^{20,45}$ The thin films made from nanorods exhibited a PLQY up to $19 \%$ and $32 \%$ at $405 \mathrm{~nm}$ and $445 \mathrm{~nm}$ excitation, respectively. Interestingly, pumping the cubic phase $\left(\lambda_{\text {exc }}=485 \mathrm{~nm}\right)$ yielded high PLQY up to $70 \%$, which is comparatively higher than the pure cubic phase. For more details, please refer S6 of SI. However, the quantum yield decreased when exciting further into the band edge since it becomes impossible to record the entire emission range, which partly overlaps with higher excitation wavelengths.
To investigate how the two different emitting species in the quasi-2 $\mathrm{D}$ conglomerate arrange themselves relative to each other, we first attempted Transmission Electron Microscope (TEM) on these nanorods. However, since the perovskites are very sensitive to high-energy probes, the TEM results were not conclusive. Therefore, we chose to conduct relatively low-energy, two-photon excitation and imaging, on these nanorods, as shown in Figure 3c. Here we have chosen to examine and compare the PL intensity maps recorded consecutively on the same collection of nanorods using both $910 \mathrm{~nm}$ and 1064 nm two-photon excitation, corresponding to excitation energies equivalent to $455 \mathrm{~nm}$ and $532 \mathrm{~nm}$. In this way, the $910 \mathrm{~nm}$ map will consist of an emission comprised of mainly blue (2D) and some green $(3 \mathrm{D})$, while the 1064 $\mathrm{nm}$ map will only excite the green emitting phase. Thus, if the two separate phases do not coincide in space, there should be an anticorrelation between the corresponding emission maps presented in Figure 3c; i.e. a region which is brighter under one excitation wavelength should be relatively weaker under the other. It follows that evaluating the intensities of corresponding pixels forming the two-photon PL maps (Figure 3d), there exists a strong void where a correlation would exists (i.e. if all points in space were to emit equivalently), and we see instead clear evidence for anticorrelation. At least on the scale of this optical diffraction limited images, the existence and separating distance of the two phases making up the nanorods can be resolved, confirming the quasi-2D microstructure separation of the different phases revealed through both our XRD refinement and optical absorption/emission studies.

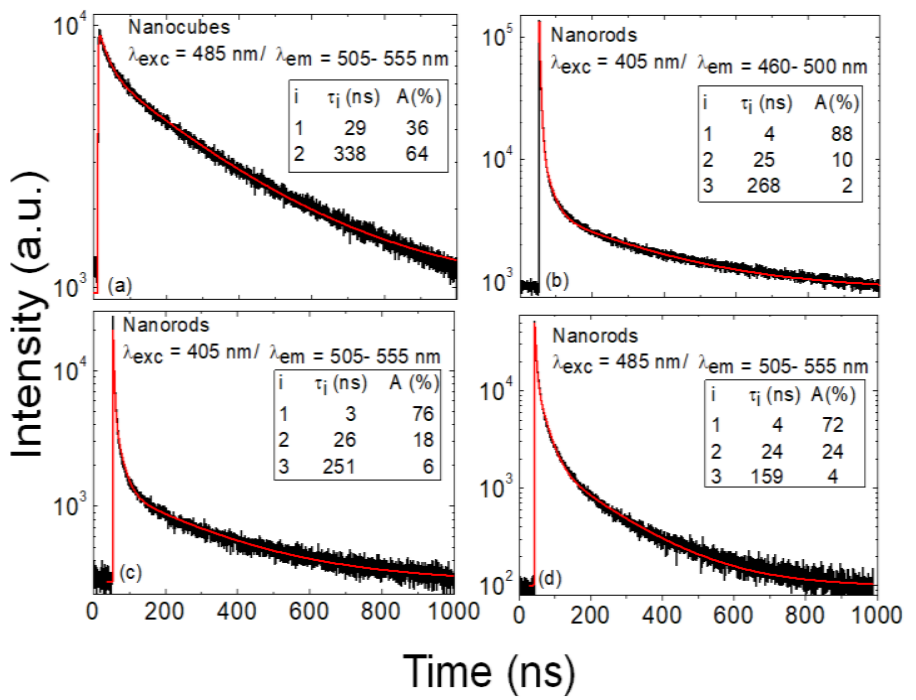

Figure 4. Fluorescence lifetime over the first microsecond for (a) $\mathrm{FAPbBr}_{3}$ nanocubes and (b)-(d) nanorods, with the experimental details and fitting (red line) parameters displayed in the insets. 

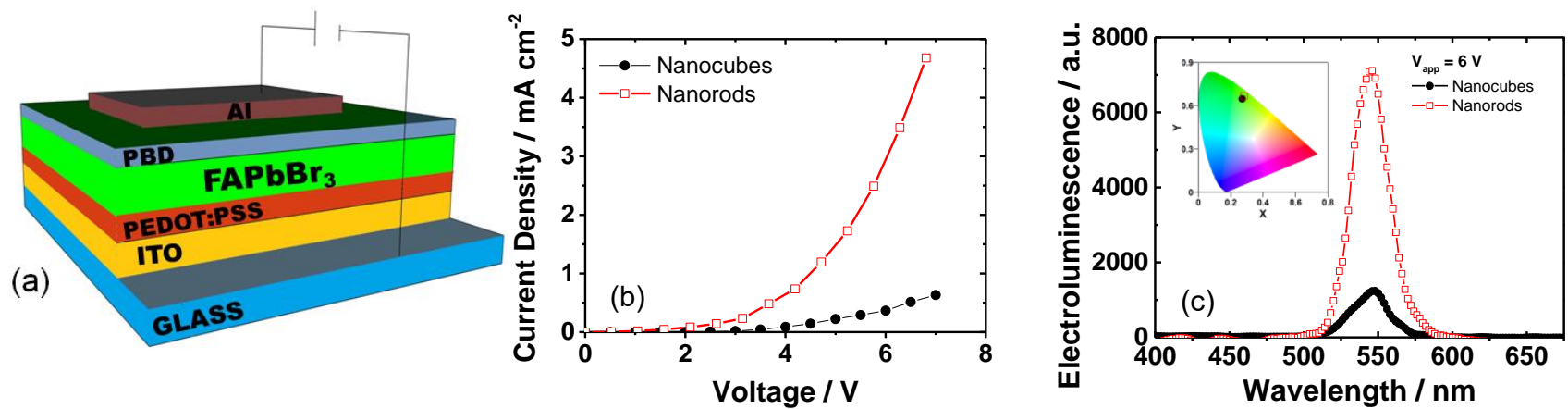

Figure 5. a) PeLED configuration for comparison of the b) I-V response curve for the $\mathrm{FAPbBr}_{3} \mathrm{PbBr}_{2} / \mathrm{FABr} 1: 1$ nanocubes (black dots) and 1:3 nanorods (red squares) and c) normalized electroluminescence spectra of the hybrid multilayer devices upon $6 \mathrm{~V}$ voltage supply. The inset in panel c) displays the CIE coordinates of the electroluminescence at $6 \mathrm{~V}$.

However, resolving phases as well as the ratio of the phases within one crystal is impossible due to diffraction limit of this technique. Furthermore, we executed an excitation power dependence study on potential structural changes and also investigated the influence of excitation power on the growth of the green-emitting phase as well as the stability of nanorods. Please refer to SI (Figure $\mathrm{S}_{7}$ and S8) for more details on this experiment.

\section{PL lifetime measurements:}

In evaluating the kinetics of photogenerated charges within our NC thin films, we next investigate the nature of radiative recombination via fluorescence lifetime imaging of an ensemble of NCs. Due to the significant overlap in the two emission bands arising from our quasi-2D nanorods, clean deconvolution of the decaying species is difficult. By assessing the decaying PL signals near each emission wavelength maxima, i.e. at $490 \mathrm{~nm}$ and at 530 $\mathrm{nm}$, we can make a qualitative evaluation of the charge dynamic in the two-phase nanorods. Typical PL decays are presented in Figure 4 and their temporal intensities, $\mathrm{I}(\mathrm{t})$, were analyzed using either a bi- or tri-exponential fit: $I(t)=\sum_{i=1}^{3} A_{i} \exp \left(\frac{-t}{\tau_{i}}\right)$. Here $\tau_{\mathrm{i}}$ represent the different lifetime components in order of an increasing time constant, corresponding with a decay amplitude $A_{i}$. Further, the lifetime data were recorded through imaging an ensemble of NCs $(-20 \times 20 \mu \mathrm{m})$, forming the basis of the averaged lifetime and amplitude values inset into Figure 4. Generally, the PL emission in perovskites can be described using a simple three-charge recombination pathway model (approximately on three different time scales): (i) fast recombination of bound excitons, or trap-assisted recombination (1-10 ns), (ii) recombination of unbounded excitons (10 - $100 \mathrm{~ns}$ ), and (iii) clean band-to-band recombination of free charge carriers (10os of ns). Photoexciting a perovskite crystal, these three processes are observed simultaneously in the PL decay and appropriately fit with three different characteristic exponential components. Among the decaying species, the recombination of excitons typically occurs fastest, and free carrier recombination takes place on much longer time scales - free electrons and holes will scatter though the crystal ${ }^{47}$ and will potentially transfer across interfacing phases, to only recombine once they overlap. First, exciting the $\mathrm{FAPbBr}_{3}$ nanocubes in Figure 4a with $485 \mathrm{~nm}$ light, two decaying species govern the detected emission at $545 \mathrm{~nm}$; an average fast recombination of 29 ns and an average long component of 338 ns (Table S2). Conversely, for the $\mathrm{FAPbBr}_{3}$ nanorods, three measurement schemes were targeted for comparison; excitation far above the two absorption edges $(405 \mathrm{~nm})$ to examine both the blue (Figure 4b) and green (Figure 4c) emitting species, and exciting close to the $3 \mathrm{D}$ absorption edge $(485) \mathrm{nm})$ to understand the decay dynamics of the green emission. Overviewing the various decay components of the nanorods, we find that all three are well-described by triexponential fits. For all datasets, the decay rate is dominated by the fast component $(\sim 79 \%)$, indicating a quantum confined quasi-2D system. Typically, for a pure $(\mathrm{OAm})_{2} \mathrm{PbBr}_{4}$ system, we obtained a monoexponential decay of 2.5 ns. (Figure $\mathrm{S}_{5} \mathrm{~d}$ ) This is unsurprising given the general constraints of excitonic recombination within a quantum confined system. Hence, we assign this fast decay to the quasi-2D component in the nanorods (Figure 4b). Interestingly, for the green emitting portion of the nanorods, two long decay components appear again. On exciting the two-phase material with $405 \mathrm{~nm}$ light, we imply an interplay of two different processes; i) direct excitation of green emitting species, ii) a substantial portion of excited carriers migrates from the crystal constituent with a wide bandgap to the other with more narrow bandgap. (Figure 4c) As previously stated, this notion has been repeatedly observed in quasi-2D perovskite systems. ${ }^{48,49}$ On the other hand, moving to $485 \mathrm{~nm}$ excitation causes the long component of the green emission to dramatically shorten to just over 159 ns (Figure $4 \mathrm{~d}$ and Table S2). This lifetime is substantially shorter than the longest component of the green emission originating from a pure cubic structure (Figure $4 \mathrm{a}$ and b). Furthermore, at $485 \mathrm{~nm}$ excitation wavelength, the emission contribution is mostly from the $3 \mathrm{D}$ perovskites, however, as we also excite the tail end of the quasi-2D blue emissive part, the fast decay component also contributes to the lifetime decay. As 
mentioned before, the XRD and PL data confirmed the dual phase nature of rods, which indicates lower crystal purity when compared to the common cubic phase, presumably leading to the faster decay. Lifetime measurements on single rods were not recorded owning to the long decay times observed and the subsequent low photon counts. A more detailed study on the complex decay behavior of the system is on the way.

\section{LED fabrication and characterization:}

Based on the good optoelectronic properties described in the previous sections, the application of cube- and rodshaped $\mathrm{FAPbBr}_{3} \mathrm{NCs}$ was tested in light emitting diode (LED) devices in order to unravel the effect of the morphology in the device performance. This type of perovskite material presents its valance band and conduction band around $\sim 3,4$ and $5.6 \mathrm{eV}$, respectively,,$^{50,51}$ which is a good approximation for the energy levels and electrodes selection when designing our device. Therefore, a double layer LED was fabricated using the following configuration: ITO anode/PEDOT:PSS ( $40 \mathrm{~nm}) / 25 \mathrm{wt} \%$ of the FAP$\mathrm{bBr}_{3} \mathrm{NCs}$ in a toluene $(220 \mathrm{~nm}) / \mathrm{p}$-PBD $(20 \mathrm{~nm}) \mid \mathrm{Al}(150$ $\mathrm{nm})$. All the layers were spincoated, besides the $\mathrm{Al}$ metal cathode $(150 \mathrm{~nm})$ which was thermally evaporated. (Figure 5a) The reason for using p-PBD is to improve the electron injection into the perovskite emissive layer and at the same time to inhibit the direct interaction between the $\mathrm{Al}$ metal and the perovskite material, which in many cases degrades the perovskite. ${ }^{52}$ Please note that currently, we did not optimize the thin film quality of these perovskites as the application is presented just as a proof-ofprinciple study. Nevertheless, the uniformity of the applied perovskite thin film layer was measured using a non-contact optical profilometer and the results are depicted in Figure Sio. Figure 5b shows the current-voltage $I-V$ curve for the $\mathrm{PbBr}_{2} / \mathrm{FABr}$ 1:1 nanocubes and 1:3 nanorods devices, where in both cases, a typical diode characteristic was obtained..$^{53}$ Both morphologies exhibit a comparable turn-on voltage of around $\sim 2.8 \mathrm{~V}$. However, it is worth to note that the current density for the nanorods is enhanced by 7 fold at the same bias. This observation suggests that the $\mathrm{FAPbBr}_{3}$ nanorods exhibit better charge injection or mobility properties than the $\mathrm{FAPbBr}_{3}$ nanocubes. To obtain further insights on the optoelectronic properties, the electroluminescence (EL) spectra were recorded (Figure $5 \mathrm{c}$ ). As it is shown in the CIE coordinates $((0.27,0.65)$ and $(0.28,0.67)$ for nanocubes and nanorods, respectively), the EL spectra features for both samples are nearly identical. However, the EL intensity for the monoclinic nanorods is increased by a factor of 6 under the same bias $(6 \mathrm{~V})$. This fact confirms that the charge transport and mobility are improved by modifying the $\mathrm{FAPbBr}_{3}$ crystal structure and morphology. Interestingly, the green EL for cubes $\left(\lambda_{\max }=547 \mathrm{~nm}, \mathrm{FWHM}=28\right.$ $\mathrm{nm})$ perfectly matches its corresponding PL spectrum (Figure 4), while only the green emission component is contained in the EL spectrum of the nanorods $\left(\lambda_{\max }=545\right.$ $\mathrm{nm}, \mathrm{FWHM}=27 \mathrm{~nm}$ ). This has been shown in previous studies and could be a consequence of either a) a higher charge injection and/or more balanced transport into the ${ }_{3} \mathrm{D}$ phase component ${ }^{54}, \mathrm{~b}$ ) a substantial contribution of radiative recombination of charges in the smallest band gap $3 \mathrm{D}$ phase $\mathrm{e}^{11}$ or c) the instability of the lower dimensional phase under heating or moist conditions.

The exact mechanism is still unknown and requires further investigation. Additionally, further steps to stabilize the monoclinic structure of $\mathrm{FAPbBr}_{3}$ perovskites are necessary to improve the device stability and performance.

\section{Conclusion}

We demonstrated a facile and scalable method to prepare $\mathrm{FAPbBr}_{3} \mathrm{NCs}_{\mathrm{Caving}}$ different morphologies and physicochemical properties by compositional engineering. By applying a three-fold higher molar ratio of $\mathrm{FABr}, \mathrm{FAPbBr}$ nanorods were formed, exhibiting a hybrid quasi-2D perovskite structure. For the first time, a monoclinic phase was observed for this type of $\mathrm{FAPbBr} 3$ nanorods. The additional quasi-2D component significantly improved the PLQY from 30 to $70 \%$. The high PLQY value could be attributed to (i) the existence of $\mathrm{FAPbBr}_{3}$ perovskite in the monoclinic phase, (ii) the quasi-2 $\mathrm{D} / 3 \mathrm{D}$ quantum well-like band alignment in the crystal structure or (iii) the excess of bromide atoms exhibiting a passivating effect to the surface defects. Furthermore, this study reveals a unique mechanism to synthesize a quasi-2D perovskite structure. The presence of a lower dimensional layer containing $\mathrm{OAmBr}$ in the nanorods prepared by a $1: 3 \mathrm{PbBr}_{2} / \mathrm{FABr}$ precursor ratio was further confirmed by XRD, EDX, XPS, and time-resolved PL microscopy. Finally, LEDs were fabricated to investigate the potential application of these $\mathrm{NCs}$ in optoelectronic devices. Both types of perovskites nanocrystals exhibited a comparable performance when employed as an emissive layer in LEDs. However, due to the exotic nature of the two-phase system, the impact of different morphologies on the device performance still remains elusive. This work offers new opportunities to study the unexplored monoclinic phase of $\mathrm{FAPbBr}_{3} \mathrm{NCs}$ and resolve important questions concerning the charge dynamics within the novel two-phase systems.

\section{Methods}

1. Experimental procedure

For the synthesis of $\mathrm{FAPbBr}_{3}$, a precursor solution consisting of $0.02 \mathrm{mmol} \mathrm{PbBr2}$ and $0.02 \mathrm{mmol}$ or $0.06 \mathrm{mmol}$ $\mathrm{FABr}$ (1:1 and 1:3 precursor ratios respectively) dissolved in $1 \mathrm{ml}$ of $\mathrm{N}$-dimethylformamide (DMF) was injected dropwise into a vigorously stirring toluene solution, containing oleylamine (OAm) and oleic acid (OAc) as capping ligands. To ensure colloidal stability, the concentration of OAc was fixed to $0.2 \mathrm{mmol}$. $0.02 \mathrm{mmol}$ and $0.04 \mathrm{mmol}$ of OAm was added for the 1:1 and 1:3 $\mathrm{PbBr} /$ FAbr precursor ratios respectively, as these concentrations yielded the 
most homogeneous NC morphology. After injection, highly luminescent NCs were rapidly formed and to further promote the precipitation/crystal growth, an extra volume of toluene was subsequently added to the suspension. The resulting NCs were centrifuged at $3200 \mathrm{rpm}$ for 15 minutes and washed with toluene to remove the excess of capping agents and unreacted precursor. Finally, they were redispersed in toluene forming a stable colloidal solution. Please refer to the experimental section in the Supporting Information for schematic representation of the synthesis procedure. (Figure $\mathrm{S} 1$ )

\section{Instruments}

2.1 Scanning Electron Microscope (SEM): Morphology and composition analysis were studied using FEI Quanta FEG250 environmental scanning electron microscope at 10 $\mathrm{KV}$, equipped with an energy-dispersive spectrometer. The nanoparticles suspension was drop-casted on a silicon chip prior to the measurement.

2.2 Synchrotron X-Ray Diffraction: Synchrotron-based Xray diffraction data were collected at BMor (SNBL/ESRF in Grenoble, France) using PILATUS@SNBL diffractometer. ${ }^{55}$ The monochromatic beam $(\lambda=0.95774 \AA)$ and the parameters of the detector were calibrated on LaB6 powder using PyFAI. ${ }^{56}$ The obtained calibrations were implemented to Bubble for further azimuthal integration of $2 \mathrm{D}$ images. The resulting unit cell models were refined using the Le Bail method in Fullprof. ${ }^{57}$

2.3 UV-Vis Diffuse reflectance Spectroscopy: Diffuse reflectance spectra (DRS) were recorded on a Perkin Elmer Lambda 950 UV-VIS-NIR spectrophotometer with the $150 \mathrm{~mm}$ integrating sphere accessory in the wavelength range between $300 \mathrm{~nm}$ to $600 \mathrm{~nm}$. The diffuse reflectance $(R)$ data were converted to $F(R)$ using the Kubelka-Monk function: $F(R)=(1-R)_{2} /(2 R)$.

2.4 Emission and Excitation Spectroscopy: Excitation and emission spectra were recorded on an Edinburgh Instruments FLS 980 spectrofluorimeter. Samples were measured in front-face mode as thin films created by dropcasting a suspension onto a glass slide that fitted into the Edinburgh solids accessory.

2.5 Photoluminescence quantum yield: Quantum yield measurements were performed on a Horiba Fluorolog 3.22 spectrofluorimeter with F-3029 integrating sphere accessory fitted with a sample holder to accommodate thin film samples drop-casted on a glass slide.

2.6 Two-photon Microscopy: The photoluminescence maps were recorded on an upright microscope (BX61WI/FV10oo, Olympus) with 25×, 1.05 NA water immersion objective (XLPLAN, Olympus) using picosecond pulsed lasers of $1064 \mathrm{~nm}$ (picoTRAIN, High-Q) and 910 nm (Levante Emerald, APE-Berlin; optical parametric oscillator synchronously pumped by the second harmonic of the Nd:YVO4 picoTRAIN laser) as excitation sources. The power of each beam at focus was $15 \mathrm{~mW}$. For both excitation sources, the photoluminescence was detected in the epi-direction and transmitted through a 495-540 $\mathrm{nm}$ band pass filter before reaching a photomultiplier tuber. The optical resolution of two-photon microscopy can be estimated by the FWHM of the point-spread function, given by equation 1 and 2 (for an objective with a numerical aperture superior to 0.7 ):

$$
\begin{aligned}
& r_{x y}=\frac{0.325 \lambda}{\sqrt{2} N A^{0.91}} 2 \sqrt{\ln (2)} \\
& r_{z}=\frac{0.532 \lambda}{\sqrt{2}}\left[\frac{1}{n-\sqrt{n^{2}-N A^{2}}}\right] 2 \sqrt{\ln (2)}
\end{aligned}
$$

Where $\lambda$ is the excitation wavelength, $\mathrm{n}$ is the refractive index of the immersion medium, and NA is the numerical aperture of the objective. Thus, as we used $910 \mathrm{~nm}$ and $1064 \mathrm{~nm}$ as the two excitation sources, the resolution for the two images are respectively given by:

$$
\begin{aligned}
& r_{x y, 910}=0.316 \mu \mathrm{m} ; r_{z, 910}=1.102 \mu \mathrm{m} \\
& r_{x y, 1064}=0.369 \mu \mathrm{m} ; r_{z, 1064}=1.289 \mu \mathrm{m}
\end{aligned}
$$

2.7 PL Lifetime spectroscopy: Fluorescence lifetime data were recorded on a home-built confocal FLIM microscope. Emission from a pulsed 405-nm laser diode (LDH$\mathrm{P}-\mathrm{C}-405$, Picoquant, Berlin, Germany) was cleaned up spectrally (Semrock 406/15 Brightline HC (AHF F37-406)). Emission from a pulsed 485-nm laser diode (LDH-D-C485, Picoquant) was cleaned up spectrally (Chroma ET485/20x, F49-482, AHF Analysentechnik, Tübingen, Germany). Laser pulsing was set to $1 \mathrm{MHz}$ (PDL 828 Sepia2, Picoquant). The laser was coupled into a singlemode polarization maintaining optical fiber (PMC-40oSi2.6-NAo12-3-APB-15o-P, Schäfter+Kirchhoff GmbH, Hamburg, Germany) using a 6oFC-4-RGBV11-47 fiber coupler (SuK). Light was collimated using a collimator with xyz adjustable lens (6oFC-L-4-RGBV11-47, SuK), the linear polarization cleaned up (CCM1-PBS251, Thorlabs $\mathrm{GmbH}$, Dachau, Germany) and light was reflected via a 3-mm thick polychroic mirror (Chroma zt405/488/561/64orpc, F73-410, AHF) into a galvanometric mirror scanner (TILL Yanus IV digital scanner, FEI Munich, Gräfelfing, Germany) that was connected to the back port of the microscope body (IX71, Olympus Belgium, Berchem Belgium). Imaging was controlled via a home-written software (C\#, Microsoft Visual Studio ${ }^{\circledast}$ ). Inside the microscope body the light was reflected upwards (3-mm thick Full Reflective Ag Mirror, F21-005, AHF, mounted in a TIRF Filter Cube for $\left.\mathrm{BX}_{2} / \mathrm{IX}_{2}, \quad \mathrm{~F} 91-960, \mathrm{AHF}\right)$ to the objective (UPLSAPO-6oXW, Olympus). Sample emission transmitted through the polychroic mirror was focussed through a $50-\mu \mathrm{m}$ pinhole $\left(\mathrm{P}_{5} \mathrm{OS}\right.$, Thorlabs) via an achromatic lens (AC254-150-A-ML, Thorlabs) and collimated again (AC254-50-A-ML, Thorlabs). After collimation, the emis- 
sion was reflected on a dichroic mirror $\left(\mathrm{H}_{5} 60 \mathrm{LPXR}, \mathrm{F}_{4} 8\right.$ 559, AHF). Then, the emission was spectrally split into two bands via an additional dichroic mirror ( $\mathrm{H}_{507} \mathrm{LPXR}$, F48-507): the blue part was spectrally filtered through a 450/5o ET Bandpass emission filter (AHF), the green part through a $\mathrm{HQ}_{525} / 50$ bandpass filter (Chroma). Emission was focused (AC254-50-A-ML, Thorlabs) on an avalanche photodiode ( $\tau$-SPAD, Picoquant). The detector was connected to a time-correlated single photon counting (TCSPC) device (Hydraharp 40o, Picoquant) and powered using a power supply (DSN-102, Picoquant). The laser powers used were $0.15,0.75$ and $1.5 \mathrm{nW}$, measured between the polychroic mirror and the galvo (LabMax Top, Coherent, Santa Clara, California, USA)(about 40\% reached the sample), corresponding to 50, 250 and 500 $\mathrm{mW} / \mathrm{cm} 2$ respectively (taking into account the size of the focal spot and percentage of laser that actually reaches the sample). Data were loaded in the PAM software. ${ }^{58}$ (https://pam.readthedocs.io) written in MATLAB (The MathWorks, Eindhoven, The Netherlands). Instrument response functions (IRF) were recorded by removing the emission filter and imaging the reflection of the laser on

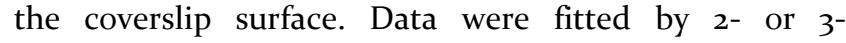
component convolution fitting.

\section{ASSOCIATED CONTENT}

This material is available free of charge via the Internet at http://pubs.acs.org.

Detailed description of synthesis procedure, instruments and results.

Size distribution of cubes and rods.

Elemental composition.

Peak analysis of XPS spectra.

SEM and XRD of $\mathrm{FAPbBr}_{3}$ formed after adding $\mathrm{HBr}$ to 1:1 $\mathrm{PbBr}_{2} / \mathrm{FABr}$.

SEM, XRD, PL, absorption and lifetime of $2 \mathrm{D}$ perovskites $(\mathrm{OAm})_{2} \mathrm{PbBr} 4$.

PL, absorption and PLQY of nanorods recorded in different atmospheric conditions.

Two photon excitation: Effect of excitation power on structural changes and stability.

Recombination lifetimes of nanocubes and nanorods.

Thin film morphology of perovskites used as emissive layer in LEDs.

\section{AUTHOR INFORMATION}

\section{Corresponding Author}

* Email: Elke.debroye@kuleuven.be

* Email: Johan.hofkens@kuleuven.be

\section{ACKNOWLEDGMENT}

The authors acknowledge financial support from the Research Foundation - Flanders (FWO Grant numbers G.oB39.15, G.oB49.15, Go98319N and ZW15_o9-GOH6316), the EC Marie Curie ITN - iSwitch PhD fellowship to H.B. (Grant number 642196), the Research Foundation - Flanders postdoctoral fellowships to J.A.S., C.M., M.K., H.Y. and E.D.
(FWO Grant numbers ${ }_{12} \mathrm{Y}_{7218 \mathrm{~N},}$ 12J1716N, $12 \mathrm{Y} 6418 \mathrm{~N}$, $12 \mathrm{R} 8718 \mathrm{~N}$ and ${ }_{12} \mathrm{O} 3719 \mathrm{~N}$, respectively), the Research Foundation - Flanders PhD fellowship to G.S.F. (FWO Grant number $1193818 \mathrm{~N})$, the KU Leuven Research Fund (C14/15/053), the Flemish government through long term structural funding Methusalem (CASAS2, Meth/15/04) and the Hercules foundation $(\mathrm{HER} / 11 / 14)$. J.A.S. and H.Y. acknowledge the XRD support provided by Vadim Diadkin on BMor at the ESRF.

\section{REFERENCES}

(1) Murali, B.; Yengel, E.; Yang, C.; Peng, W.; Alarousu, E.; Bakr, O. M.; Mohammed, O. F. The Surface of Hybrid Perovskite Crystals: A Boon or Bane. ACS Energy Letters 2017, 2 (4), 846856.

(2) Jeon, N. J.; Noh, J. H.; Yang, W. S.; Kim, Y. C.; Ryu, S.; Seo, J.; Seok, S. I. Compositional Engineering of Perovskite Materials for High-Performance Solar Cells. Nature 2015, 517 (7535), 476-48o.

(3) Kim, Y.-H.; Cho, H.; Heo, J. H.; Kim, T.-S.; Myoung, N.; Lee, C.-L.; Im, S. H.; Lee, T.-W. Multicolored Organic/Inorganic Hybrid Perovskite Light-Emitting Diodes. Advanced Materials 2015, 27 (7), 1248-1254.

(4) Cho, H.; Jeong, S.-H.; Park, M.-H.; Kim, Y.-H.; Wolf, C.; Lee, C.-L.; Heo, J. H.; Sadhanala, A.; Myoung, N.; Yoo, S.; et al. Overcoming the Electroluminescence Efficiency Limitations of Perovskite Light-Emitting Diodes. Science 2015, 350 (6265), 12221225 .

(5) Tan, Z.-K.; Moghaddam, R. S.; Lai, M. L.; Docampo, P.; Higler, R.; Deschler, F.; Price, M.; Sadhanala, A.; Pazos, L. M.; Credgington, D.; et al. Bright Light-Emitting Diodes Based on Organometal Halide Perovskite. Nature Nanotechnology 2014, 9 (9), 687-692.

(6) Zhao, Y.; Zhu, K. Organic-Inorganic Hybrid Lead Halide Perovskites for Optoelectronic and Electronic Applications. Chemical Society Reviews 2016, 45 (3), 655-689.

(7) Huang, H.; Zhao, F.; Liu, L.; Zhang, F.; Wu, X.; Shi, L.; Zou, B.; Pei, Q.; Zhong, H. Emulsion Synthesis of Size-Tunable $\mathrm{CH}_{3} \mathrm{NH}_{3} \mathrm{PbBr}_{3}$ Quantum Dots: An Alternative Route toward Efficient Light-Emitting Diodes. ACS Appl. Mater. Interfaces 2015, 7 (51), 28128-28133.

(8) Lee, J.-W.; Choi, Y. J.; Yang, J.-M.; Ham, S.; Jeon, S. K.; Lee, J. Y.; Song, Y.-H.; Ji, E. K.; Yoon, D.-H.; Seo, S.; et al. In-Situ Formed Type I Nanocrystalline Perovskite Film for Highly Efficient Light-Emitting Diode. ACS Nano 2017, 11 (3), 3311-3319.

(9) Bohn, B. J.; Tong, Y.; Gramlich, M.; Lai, M. L.; Döblinger, M.; Wang, K.; Hoye, R. L. Z.; Müller-Buschbaum, P.; Stranks, S. D.; Urban, A. S.; et al. Boosting Tunable Blue Luminescence of Halide Perovskite Nanoplatelets through Postsynthetic Surface Trap Repair. Nano Lett. 2o18, 18 (8), 5231-5238.

(10) Lignos, I.; Protesescu, L.; Emiroglu, D. B.; Maceiczyk, R.; Schneider, S.; Kovalenko, M. V.; deMello, A. J. Unveiling the Shape Evolution and Halide-Ion-Segregation in Blue-Emitting Formamidinium Lead Halide Perovskite Nanocrystals Using an Automated Microfluidic Platform. Nano Lett. 2018, 18 (2), 12461252.

(11) Yang, X.; Zhang, X.; Deng, J.; Chu, Z.; Jiang, Q.; Meng, J.; Wang, P.; Zhang, L.; Yin, Z.; You, J. Efficient Green LightEmitting Diodes Based on Quasi-Two-Dimensional Composition and Phase Engineered Perovskite with Surface Passivation. Nature Communications 2018, 9 (1).

(12) Kumar, S.; Jagielski, J.; Yakunin, S.; Rice, P.; Chiu, Y.-C.; Wang, M.; Nedelcu, G.; Kim, Y.; Lin, S.; Santos, E. J. G.; et al. Efficient Blue Electroluminescence Using Quantum-Confined 
Two-Dimensional Perovskites. ACS Nano 2016, 10 (10), 97209729.

(13) Li, Z.; Yang, M.; Park, J.-S.; Wei, S.-H.; Berry, J. J.; Zhu, K. Stabilizing Perovskite Structures by Tuning Tolerance Factor: Formation of Formamidinium and Cesium Lead Iodide SolidState Alloys. Chemistry of Materials 2016, 28 (1), 284-292.

(14) Yuan, H.; Debroye, E.; Janssen, K.; Naiki, H.; Steuwe, C.; Lu, G.; Moris, M.; Orgiu, E.; Uji-i, H.; De Schryver, F.; et al. Degradation of Methylammonium Lead Iodide Perovskite Structures through Light and Electron Beam Driven Ion Migration. The Journal of Physical Chemistry Letters 2016, 7 (3), 561-566.

(15) Xiao, Z.; Kerner, R. A.; Zhao, L.; Tran, N. L.; Lee, K. M.; Koh, T.-W.; Scholes, G. D.; Rand, B. P. Efficient Perovskite LightEmitting Diodes Featuring Nanometre-Sized Crystallites. Nature Photonics 2017, 11 (2), 108-115.

(16) Quan, L. N.; Yuan, M.; Comin, R.; Voznyy, O.; Beauregard, E. M.; Hoogland, S.; Buin, A.; Kirmani, A. R.; Zhao, K.; Amassian, A.; et al. Ligand-Stabilized Reduced-Dimensionality Perovskites. Journal of the American Chemical Society 2016, 138 (8), 2649-2655.

(17) Aharon, S.; Etgar, L. Two Dimensional Organometal Halide Perovskite Nanorods with Tunable Optical Properties. Nano Lett. 2016, 16 (5), 3230-3235.

(18) Chen, Y.; Sun, Y.; Peng, J.; Tang, J.; Zheng, K.; Liang, Z. 2D Ruddlesden-Popper Perovskites for Optoelectronics. Advanced Materials 2018, 30 (2), 1703487.

(19) Li, L.; Zhou, N.; Chen, Q.; Shang, Q.; Zhang, Q.; Wang, X.; Zhou, H. Unraveling the Growth of Hierarchical Quasi-2D/3D Perovskite and Carrier Dynamics. J. Phys. Chem. Lett. 2018, 9 (5), 1124-1132.

(20) Byun, J.; Cho, H.; Wolf, C.; Jang, M.; Sadhanala, A.; Friend, R. H.; Yang, H.; Lee, T.-W. Efficient Visible Quasi-2D Perovskite Light-Emitting Diodes. Advanced Materials 2016, 28 (34), 7515-7520.

(21) Wang, Z.; Wang, F.; Sun, W.; Ni, R.; Hu, S.; Liu, J.; Zhang, B.; Alsaed, A.; Hayat, T.; Tan, Z. Manipulating the Tradeoff Between Quantum Yield and Electrical Conductivity for High-Brightness Quasi-2D Perovskite Light-Emitting Diodes. Advanced Functional Materials 2018, 28 (47), 1804187.

(22) Zhang, F.; Zhong, H.; Chen, C.; Wu, X.; Hu, X.; Huang, H.; Han, J.; Zou, B.; Dong, Y. Brightly Luminescent and ColorTunable Colloidal $\mathrm{CH}_{3} \mathrm{NH}_{3} \mathrm{PbX}_{3}(\mathrm{X}=\mathrm{Br}, \mathrm{I}, \mathrm{Cl})$ Quantum Dots: Potential Alternatives for Display Technology. ACS Nano 2015, 9 (4), 4533-4542.

(23) Huang, H.; Susha, A. S.; Kershaw, S. V.; Hung, T. F.; Rogach, A. L. Control of Emission Color of High Quantum Yield $\mathrm{CH}_{3} \mathrm{NH}_{3} \mathrm{PbBr}_{3}$ Perovskite Quantum Dots by Precipitation Temperature. Advanced Science 2015, 2 (9), 1500194.

(24) Zhang, F.; Chen, C.; Kershaw, S. V.; Xiao, C.; Han, J.; Zou, B.; Wu, X.; Chang, S.; Dong, Y.; Rogach, A. L.; et al. LigandControlled Formation and Photoluminescence Properties of $\mathrm{CH}_{3} \mathrm{NH}_{3} \mathrm{PbBr}_{3}$ Nanocubes and Nanowires. ChemNanoMat 2017, 3 (5), 303-310.

(25) Quan, L. N.; Zhao, Y.; García de Arquer, F. P.; Sabatini, R.; Walters, G.; Voznyy, O.; Comin, R.; Li, Y.; Fan, J. Z.; Tan, H.; et al. Tailoring the Energy Landscape in Quasi-2D Halide Perovskites Enables Efficient Green-Light Emission. Nano Letters 2017, 17 (6), 3701-3709.

(26) Bhaumik, S.; Veldhuis, S. A.; Ng, Y. F.; Li, M.; Muduli, S. K.; Sum, T. C.; Damodaran, B.; Mhaisalkar, S.; Mathews, N. Highly Stable, Luminescent Core-Shell Type MethylammoniumOctylammonium Lead Bromide Layered Perovskite Nanoparticles. Chem. Commun. 2016, 52 (44), 7118-7121.
(27) Debroye, E.; Yuan, H.; Bladt, E.; Baekelant, W.; Van der Auweraer, M.; Hofkens, J.; Bals, S.; Roeffaers, M. B. J. Facile Morphology-Controlled Synthesis of Organolead Iodide Perovskite Nanocrystals Using Binary Capping Agents. ChemNanoMat 2017, 3 (4), 223-227.

(28) Vassilakopoulou, A.; Papadatos, D.; Zakouras, I.; Koutselas, I. Mixtures of Quasi-Two and Three Dimensional Hybrid Organic-Inorganic Semiconducting Perovskites for Single Layer LED. Journal of Alloys and Compounds 2017, 692, 589-598.

(29) Vassilakopoulou, A.; Papadatos, D.; Koutselas, I. Room Temperature Light Emitting Diode Based on 2D Hybrid OrganicInorganic Low Dimensional Perovskite Semiconductor. Applied Materials Today 2016, 5, 128-133.

(30) Hanusch, F. C.; Wiesenmayer, E.; Mankel, E.; Binek, A.; Angloher, P.; Fraunhofer, C.; Giesbrecht, N.; Feckl, J. M.; Jaegermann, W.; Johrendt, D.; et al. Efficient Planar Heterojunction Perovskite Solar Cells Based on Formamidinium Lead Bromide. The Journal of Physical Chemistry Letters 2014, 5 (16), 27912795 .

(31) Noh, J. H.; Im, S. H.; Heo, J. H.; Mandal, T. N.; Seok, S. I. Chemical Management for Colorful, Efficient, and Stable Inorganic-Organic Hybrid Nanostructured Solar Cells. Nano Letters 2013, 13 (4), 1764-1769.

(32) Carpenter, M. A.; Howard, C. J. Symmetry Rules and Strain/Order-Parameter Relationships for Coupling between Octahedral Tilting and Cooperative Jahn-Teller Transitions in $\mathrm{ABX}_{3}$ Perovskites. I. Theory. Acta Cryst B 2009, 65 (2), 134-146.

(33) Safdari, M.; H. Svensson, P.; Tam Hoang, M.; Oh, I.; Kloo, L.; M. Gardner, J. Layered 2D Alkyldiammonium Lead Iodide Perovskites: Synthesis, Characterization, and Use in Solar Cells. Journal of Materials Chemistry A 2016, 4 (40), 15638-15646.

(34) Glazer, A. M. The Classification of Tilted Octahedra in Perovskites. Acta Crystallographica Section B Structural Crystallography and Crystal Chemistry 1972, 28 (11), 3384-3392.

(35) Weidman, M. C.; Seitz, M.; Stranks, S. D.; Tisdale, W. A. Highly Tunable Colloidal Perovskite Nanoplatelets through Variable Cation, Metal, and Halide Composition. ACS Nano 2016, 10 (8), 7830-7839.

(36) Kim, Y.-H.; Lee, G.-H.; Kim, Y.-T.; Wolf, C.; Yun, H. J.; Kwon, W.; Park, C. G.; Lee, T.-W. High Efficiency Perovskite Light-Emitting Diodes of Ligand-Engineered Colloidal Formamidinium Lead Bromide Nanoparticles. Nano Energy 2017, 38, 51-58.

(37) Milosavljević, A. R.; Huang, W.; Sadhu, S.; Ptasinska, S. Low-Energy Electron-Induced Transformations in Organolead Halide Perovskite. Angewandte Chemie International Edition 2016, 55 (34), 10083-10087.

(38) Zhang, Y.; Wang, C.; Deng, Z. Colloidal Synthesis of Monolayer-Thick Formamidinium Lead Bromide Perovskite Nanosheets with a Lateral Size of Micrometers. Chemical Communications 2018, 54 (32), 4021-4024.

(39) Huang, W.; Manser, J. S.; Sadhu, S.; Kamat, P. V.; Ptasinska, S. Direct Observation of Reversible Transformation of $\mathrm{CH}_{3} \mathrm{NH}_{3} \mathrm{PbI}_{3}$ and $\mathrm{NH}_{4} \mathrm{PbI}_{3}$ Induced by Polar Gaseous Molecules. J. Phys. Chem. Lett. 2016, 7 (24), 5068-5073.

(40) Li, X.; Wu, Y.; Zhang, S.; Cai, B.; Gu, Y.; Song, J.; Zeng, H. CsPbX 3 Quantum Dots for Lighting and Displays: RoomTemperature Synthesis, Photoluminescence Superiorities, Underlying Origins and White Light-Emitting Diodes. Advanced Functional Materials 2016, 26 (15), 2435-2445.

(41) Coetzee, J. F.; Padmanabhan, G. R. Properties of Bases in Acetonitrile as Solvent. IV. Proton Acceptor Power and Homoconjugation of Mono- and Diamines. J. Am. Chem. Soc. 1965, 87 (22), 5005-5010. 
(42) Weidman, M. C.; Goodman, A. J.; Tisdale, W. A. Colloidal Halide Perovskite Nanoplatelets: An Exciting New Class of Semiconductor Nanomaterials. Chem. Mater. 2017, 29 (12), 50195030.

(43) Almeida, G.; Goldoni, L.; Akkerman, Q.; Dang, Z.; Khan, A. H.; Marras, S.; Moreels, I.; Manna, L. Role of Acid-Base Equilibria in the Size, Shape, and Phase Control of Cesium Lead Bromide Nanocrystals. ACS Nano 2018, 12 (2), 1704-1711.

(44) Eperon, G. E.; Stranks, S. D.; Menelaou, C.; Johnston, M. B.; Herz, L. M.; Snaith, H. J. Formamidinium Lead Trihalide: A Broadly Tunable Perovskite for Efficient Planar Heterojunction Solar Cells. Energy \& Environmental Science 2014, 7 (3), 982.

(45) US20170321117A1 - Highly tunable colloidal perovskite nanoplatelets - Google Patents https://patents.google.com/patent/US20170321117A1/en (accessed Oct 31, 2018).

(46) Even, J.; Pedesseau, L.; Katan, C. Understanding Quantum Confinement of Charge Carriers in Layered 2D Hybrid Perovskites. ChemPhysChem 2014, 15 (17), 3733-3741.

(47) Shi, D.; Adinolfi, V.; Comin, R.; Yuan, M.; Alarousu, E.; Buin, A.; Chen, Y.; Hoogland, S.; Rothenberger, A.; Katsiev, K.; et al. Low Trap-State Density and Long Carrier Diffusion in Organolead Trihalide Perovskite Single Crystals. Science 2015, 347 (6221), 519-522.

(48) Milot, R. L.; Sutton, R. J.; Eperon, G. E.; Haghighirad, A. A.; Martinez Hardigree, J.; Miranda, L.; Snaith, H. J.; Johnston, M. B.; Herz, L. M. Charge-Carrier Dynamics in 2D Hybrid MetalHalide Perovskites. Nano Letters 2016, 16 (11), 7001-7007.

(49) Blancon, J.-C.; Tsai, H.; Nie, W.; Stoumpos, C. C.; Pedesseau, L.; Katan, C.; Kepenekian, M.; Soe, C. M. M.; Appavoo, K.; Sfeir, M. Y.; et al. Extremely Efficient Internal Exciton Dissociation through Edge States in Layered 2D Perovskites. Science 2017, 355 (6331), 1288-1292.

(50) Perumal, A.; Shendre, S.; Li, M.; Tay, Y. K. E.; Sharma, V. K.; Chen, S.; Wei, Z.; Liu, Q.; Gao, Y.; Buenconsejo, P. J. S.; et al. High Brightness Formamidinium Lead Bromide Perovskite

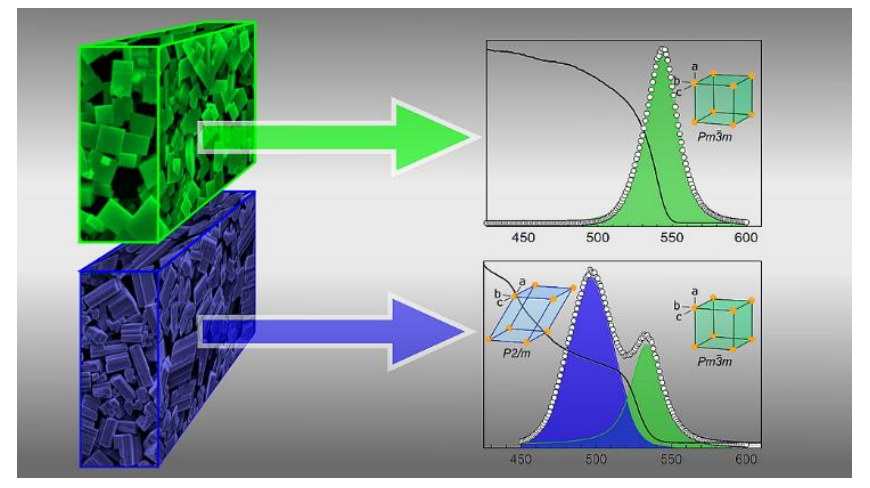

Nanocrystal Light Emitting Devices. Scientific Reports 2016, 6, 36733.

(51) Aygüler, M. F.; Weber, M. D.; Puscher, B. M. D.; Medina, D. D.; Docampo, P.; Costa, R. D. Light-Emitting Electrochemical Cells Based on Hybrid Lead Halide Perovskite Nanoparticles. The Journal of Physical Chemistry C 2015, 119 (21), 12047-12054.

(52) Guerrero, A.; You, J.; Aranda, C.; Kang, Y. S.; GarciaBelmonte, G.; Zhou, H.; Bisquert, J.; Yang, Y. Interfacial Degradation of Planar Lead Halide Perovskite Solar Cells. ACS Nano 2016, $10(1), 218-224$.

(53) Sah, C.; Noyce, R. N.; Shockley, W. Carrier Generation and Recombination in P-N Junctions and P-N Junction Characteristics. Proceedings of the IRE 1957, 45 (9), 1228-1243.

(54) Veldhuis, S. A.; Boix, P. P.; Yantara, N.; Li, M.; Sum, T. C.; Mathews, N.; Mhaisalkar, S. G. Perovskite Materials for LightEmitting Diodes and Lasers. Advanced Materials 2016, 28 (32), 6804-6834.

(55) Dyadkin, V.; Pattison, P.; Dmitriev, V.; Chernyshov, D. A New Multipurpose Diffractometer PILATUS@SNBL. Journal of Synchrotron Radiation 2016, 23 (3), 825-829.

(56) Ashiotis, G.; Deschildre, A.; Nawaz, Z.; Wright, J. P.; Karkoulis, D.; Picca, F. E.; Kieffer, J. The Fast Azimuthal Integration Python Library: PyFAI. J Appl Crystallogr 2015, 48 (2), 510519 .

(57) Rodríguez-Carvajal, J. Recent Advances in Magnetic Structure Determination by Neutron Powder Diffraction. Physica B: Condensed Matter 1993, 192 (1-2), 55-69.

(58) Schrimpf, W.; Barth, A.; Hendrix, J.; Lamb, D. C. PAM: A Framework for Integrated Analysis of Imaging, SingleMolecule, and Ensemble Fluorescence Data. Biophysical Journal 2018, 114 (7), 1518-1528. 